\title{
Stability Analysis and Control of Bifurcations of Parallel Connected DC/DC Converters Using the Monodromy Matrix
}

\author{
Abdulmajed Elbkosh, Damian Giaouris, \\ Volker Pickert and Bashar Zahawi \\ School of Electrical, Electronic and Computer \\ Engineering, Newcastle University, \\ Newcastle upon Tyne, UK \\ Email:\{Damian.Giaouris@ncl.ac.uk\}
}

\author{
Soumitro Banerjee \\ Centre for Theoretical Studies and Department of \\ Electrical Engineering, Indian Institute of Technology, \\ Kharagpur, India \\ Email: \{soumitro@ee.iitkgp.ernet.in\}
}

\begin{abstract}
The paper studies the stability of parallel DC/DC converters using the concept of monodromy matrix (the state transition matrix for one complete cycle), whose eigenvalues are the Floquet multipliers. This matrix is composed of the state transition matrices for the smooth intervals and those across the switching events (called saltation matrices). We show that instabilities in this system can be caused by smooth as well as nonsmooth period doubling bifurcations, the latter occurring when the fundamental solution matrix undergoes a discontinuous jump as a periodic solution touches a nonsmooth hyper-surface of discontinuity. Based on the expression for the saltation matrices (the state transition matrices across switching events) we propose new controllers that can stabilize the period-1 operation by keeping the eigenvalues inside the unit circle.
\end{abstract}

\section{INTRODUCTION}

Parallel operation of DC/DC converters is widely used in many applications [1], [2]. The scheme has many advantages compared to single dc-dc converters in terms of reliability, fault tolerance, and allowing high load currents to be delivered without employing devices of high power rating. Usually, it is desired to distribute the load equally among the converters [2]. One widely used method for balancing the currents is the master-slave current sharing technique employed in this paper. However, these closedloop converters are inherently nonlinear and nonsmooth time varying systems, and one can expect nonlinear phenomena in such systems.

The analysis of these phenomena is of great importance since small changes in the system's parameters (for example the supply voltage) can destabilize the system [4], [5]. However, to analyze the stability of these switching systems, one has to deal first with their discontinuity. The most common method of studying the stability of periodic systems is the Poincare map. The system's stability is indicated by the eigenvalues of the map computed at the fixed point. However, in most power electronics circuits (like the system under study) it is not possible to derive this map analytically.

In our earlier publications [4], [5], we have shown that the concept of monodromy matrix can be fruitfully applied to calculate the stability of a single dc-dc converter. The monodromy matrix is the state transition matrix over a complete clock cycle, which is a product of the state transition matrices for the $\mathrm{ON}$ and OFF periods, and those across switching events (called saltation matrices). We have also shown that the saltation matrix is primarily responsible for the stability of the system. In this paper we extend the use of the monodromy matrix to study the instabilities in parallel connected converters and we show that the fundamental solution matrix can jump if a periodic solution touches a nonsmooth hyper-surface of discontinuity. Using the expression of the saltation matrix, we propose novel controllers that can avoid instabilities.

\section{SYSTEM DISCRIPTION AND OPERATION}

Fig. 1 shows a simplified block diagram that describes the master-slave current sharing schemes for two converters connected in parallel and feeding the same load. One of the converters (the master) has a simple feedback loop (proportional gain) to regulate the output voltage. The control signal of this converter is equal to:

$$
\mathrm{V}_{\text {con } 1}(t)=V_{\text {offset }}-K_{p_{1}}\left(V_{\text {out }}(t)-V_{\text {ref }}\right)
$$

where $V_{\text {offset }}$ is the dc offset voltage needed to give a steady state duty cycle, $K_{p l}$ is the propositional gain, and $V_{r e f}$ is the reference voltage.

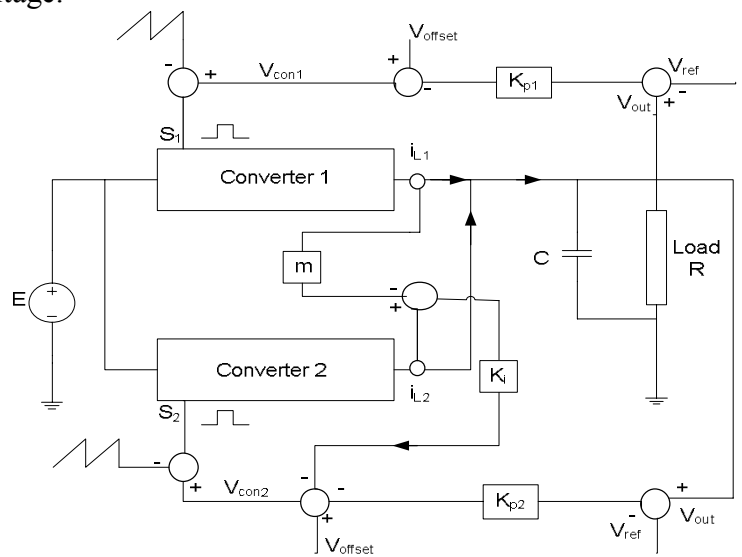

Figure 1. Block diagram of two paralleled converters with master-slave current sharing.

The other converter is the slave, which sets its current to be equal to that of the master via an active loop involving a comparison of the currents of the two converters. We can write the control signal of this converter as:

$$
\mathrm{V}_{\text {con2 }}(t)=V_{\text {offset }}-K_{p_{2}}\left(V_{\text {out }}(t)-V_{\text {ref }}\right)-K_{i}\left(i_{L_{2}}-m i_{L_{1}}\right)
$$

Where $K_{p 2}$ is the propositional gain of converter $2, K_{i}$ is the current feedback gain, and $m$ is a current factor.

The switches $S_{1}$ and $S_{2}$ are controlled by comparators which compare the control signals $V_{c o n}$ and $V_{c o n 2}$ with a suitable periodic 
saw-tooth waveform $V_{\text {ramp }}$ and a commutation occurs when these two signals become equal i.e., switch $\mathrm{S}$ is open if $V_{\text {con }}<V_{\text {ramp, }}$, and closed otherwise. The ramp signal can be written as

$$
\mathrm{V}_{\text {ramp }}(t)=V_{L}+\left(V_{U}-V_{L}\right)\left(\frac{t}{T} \bmod 1\right)
$$

where, $V_{U}$ and $V_{L}$ are the upper and lower limits of the ramp signal respectively. When the converter is operated in continuous conduction mode, there are four switch states [2]. The state equations that represent these switch states during a switching cycle are:

$$
\begin{array}{ll}
\dot{\mathbf{x}}=\mathbf{A}_{1} \mathbf{x}+\mathbf{B}_{1} \mathrm{E} & \mathrm{S}_{1} \text { ON and } \mathrm{S}_{2} \text { ON } \\
\dot{\mathbf{x}}=\mathbf{A}_{2} \mathbf{x}+\mathbf{B}_{2} \mathrm{E} & \mathrm{S}_{1} \text { ON and } \mathrm{S}_{2} \text { OFF } \\
\dot{\mathbf{x}}=\mathbf{A}_{3} \mathbf{x}+\mathbf{B}_{3} \mathrm{E} & \mathrm{S}_{1} \text { OFF and } \mathrm{S}_{2} \text { ON } \\
\dot{\mathbf{x}}=\mathbf{A}_{4} \mathbf{x}+\mathbf{B}_{4} \mathrm{E} & \mathrm{S}_{1} \text { OFF and } \mathrm{S}_{2} \text { OFF }
\end{array}
$$

Where $\mathbf{x}$ is the state vector defined as $\mathbf{x}=\left[v_{\text {out }} i_{L_{1}} i_{L_{2}}\right]^{T}=$ $\left[\begin{array}{lll}x_{1} & x_{2} & x_{3}\end{array}\right]^{T}, E$ is the input voltage and $\mathbf{A}, \mathbf{B}$ are the system matrices.

In the case of the buck converter, the system matrices $\mathbf{A}_{1}, \mathbf{A}_{2}$, $\mathbf{A}_{\mathbf{3}}$ and $\mathbf{A}_{\mathbf{4}}$ are equal to $\mathbf{A}_{\mathbf{s}}$, given by

$$
\begin{aligned}
\mathbf{A}_{s} & =\left[\begin{array}{ccc}
\frac{-1}{C R} & \frac{1}{C} & \frac{1}{C} \\
\frac{-1}{L_{1}} & \frac{-r_{L 1}}{L_{1}} & 0 \\
\frac{-1}{L_{2}} & 0 & \frac{-r_{L 2}}{L_{2}}
\end{array}\right] \text {, also } \\
\mathbf{B}_{1} & =\left[\begin{array}{lll}
0 & \frac{1}{L_{1}} & \frac{1}{L_{2}}
\end{array}\right]^{T}, \mathbf{B}_{2}=\left[\begin{array}{lll}
0 & \frac{1}{L_{1}} & 0
\end{array}\right]^{T} \\
\mathbf{B}_{3} & =\left[\begin{array}{lll}
0 & 0 & \frac{1}{L_{2}}
\end{array}\right]^{T} \text { and } \mathbf{B}_{4}=\left[\begin{array}{lll}
0 & 0 & 0
\end{array}\right]^{T}
\end{aligned}
$$

The circuit parameters are fixed at the following values: $V_{r e f}=$ $24 \mathrm{~V}, K_{p 1}=K_{p 2}=3.5, K_{i}=5, V_{\text {offset }}=5 \mathrm{~V}, L_{l}=0.02 \mathrm{H}, r_{L I}=0.05 \Omega$ $L_{2}=0.04 \mathrm{H}, r_{L 2}=0.2 \Omega, C=47 \mu \mathrm{F}, R=10 \Omega, T=400 \mu \mathrm{s}, V_{L}=2 \mathrm{~V}$, $V_{U}=8 \mathrm{~V}$.

It is worth mentioning here that since $r_{L 2}$ is greater than $r_{L 1}$, we expect the duty cycle of converter $2\left(d_{2}\right)$ to be always greater than the duty cycle of converter $1\left(d_{1}\right)$. Hence we will omit the second equation in (4) from our analysis [2].

\section{BIFURCATION BEHAVIOUR}

Like many switched dynamical systems, parallel connected converters can exhibit a variety of bifurcations. Most standard bifurcations (such as period doubling bifurcation) are caused by the change of stability status without change in topological sequence. Additionally, in switching systems, another type of bifurcation is observed, known as a border collision caused by the change of topological sequence within a clock period [1], [3]. In this system, with variation in the supply voltage it is possible to have a smooth period doubling bifurcation as shown in Fig. 2. The system loses stability to a new limit cycle with a period twice the period of the ramp signal. This bifurcation is very common in $\mathrm{dc} / \mathrm{dc}$ converters and is often considered as a standard type of bifurcation in nonlinear systems [3].

Another type of bifurcation is observed in the system by variation of the parameter $m$ (the sharing current ratio). This phenomenon is a border collision period doubling bifurcation as shown in Fig. 3. This non-smooth bifurcation occurs when the periodic solution of the system touches the non-smooth hypersurface (the top or bottom of the ramp signal) [6], [7].

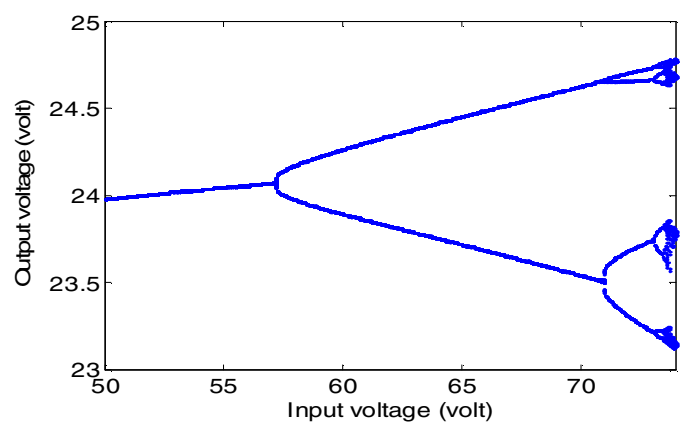

Figure 2. Bifurcation diagram with input voltage as bifurcation parameter

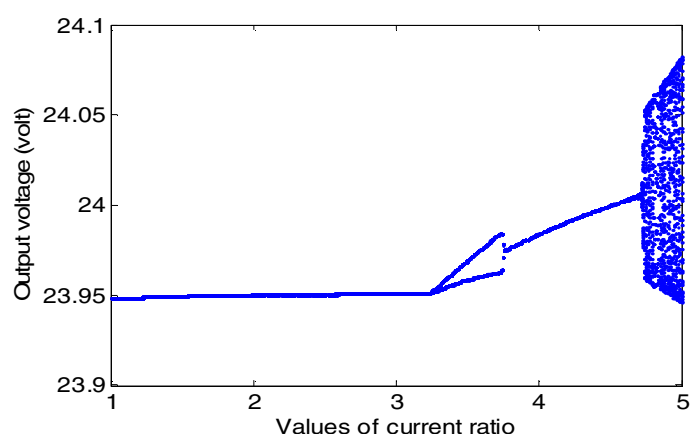

Figure 3. Bifurcation diagram with current ratio as bifurcation parameter

\section{STABILITY ANALYSIS OF THE SYSTEM}

In this section, the stability of the standard period doubling bifurcation and the border collision bifurcation are analysed by deriving the monodromy matrix of the system in each case.

\section{A. Standard bifurcation analysis:}

The period-1 limit cycle of the parallel connected buck converter given in Fig. 4 shows that the trajectory crosses the switching manifold three times at $d_{1} T, d_{2} T$ and $T$. The monodromy matrix of the system $(\mathbf{M})$ for one complete cycle is obtained as:

$$
\begin{array}{r}
\mathbf{M}(0, X(0), T)=e^{\mathbf{A}_{s}\left(1-d_{2}\right) T} \times \mathbf{S}_{\mathbf{2}} \times e^{\mathbf{A}_{s}\left(d_{2}-d_{1}\right) T} \times \mathbf{S}_{\mathbf{1}} \\
\times e^{\mathbf{A}_{\mathbf{s}} d_{1} T} \times \mathbf{S}_{\mathbf{3}}
\end{array}
$$

where $\mathbf{S}_{\mathbf{3}}, \mathbf{S}_{\mathbf{2}}$ and $\mathbf{S}_{\mathbf{1}}$ are the saltation matrices at $T, d_{2} T$ and $d_{1} T$ respectively, calculated using the following formula [4], [6]:

$$
\mathbf{S}=\mathbf{I}+\frac{\left(\mathbf{f}_{+}\left(x\left(t_{\Sigma}\right), t_{\Sigma}\right)-\mathbf{f}_{-}\left(x\left(t_{\Sigma}\right), t_{\Sigma}\right)\right) \mathbf{n}^{T}}{\mathbf{n}^{T} \mathbf{f}_{-}\left(x\left(t_{\Sigma}\right), t_{\Sigma}\right)+\frac{\partial h}{\partial t}\left(x\left(t_{\Sigma}\right), t_{\Sigma}\right)}
$$

where $h$ is the switching condition, $\mathbf{n}$ is the normal to $h, t_{\Sigma}$ is the switching time, $\mathbf{f}_{-}$and $\mathbf{f}_{+}$are the two vector fields before and after the switching.

Firstly, at $t_{\Sigma}=d_{1} T$, the switching condition is defined by $h\left(X\left(d_{1} T\right)\right)=V_{\text {offset }}-K_{p 1}\left(x_{1}\left(d_{1} T\right)-V_{\text {ref }}\right)-V_{\text {ramp }}=0$ and the normal $\mathbf{n}$ is given by $\left[\begin{array}{lll}-K_{p_{1}} & 0 & 0\end{array}\right]^{T}$. The two smooth vector fields are $\quad \mathbf{f}_{-}\left(x\left(d_{1} T\right)\right)=\mathbf{A}_{\mathbf{s}} \mathbf{x}+\mathbf{B}_{\mathbf{1}} E \quad$ and $\quad \mathbf{f}_{+}\left(x\left(d_{1} T\right)\right)=\mathbf{A}_{\mathbf{s}} \mathbf{x}+\mathbf{B}_{\mathbf{3}} E$. Since $\mathbf{f}_{+} \neq \mathbf{f}_{-}$, the system has a discontinuous vector field at the switching surface. 


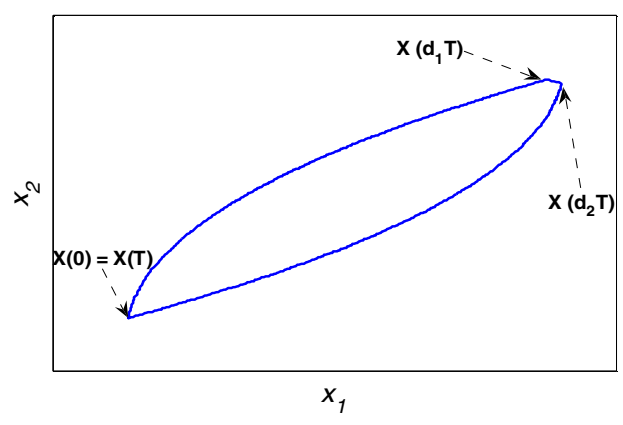

Figure 4. Period one orbit of the system for $m=1$

Secondly, at time $t_{\Sigma}=d_{2} T$, the indicator function $h\left(X\left(d_{2} T\right)\right)$ is given by the equation

$V_{\text {offset }}-K_{p_{2}}\left(x_{1}\left(d_{2} T\right)-V_{\text {ref }}\right)-K i\left(x_{3}\left(d_{2} T\right)-m x_{2}\left(d_{2} T\right)\right)-V_{\text {ramp }}=0$, $\mathbf{n}=\left[\begin{array}{lll}-K_{p_{2}} & m K_{i} & -K_{i}\end{array}\right]^{T}$ and the two smooth vector fields are given by $\mathbf{f}_{-}\left(x\left(d_{2} T\right)\right)=\mathbf{A}_{\mathbf{s}} \mathbf{x}+\mathbf{B}_{\mathbf{3}} E$ and $\mathbf{f}_{+}\left(x\left(d_{2} T\right)\right)=\mathbf{A}_{\mathbf{s}} \mathbf{x}+\mathbf{B}_{4} E$.

Finally, at $t_{\Sigma}=T$ the manifold is discontinuous (with respect to time) and the time derivative will be infinite. Therefore the saltation matrix at this point will be the identity matrix [4], [5].

The stability of the system can be determined by finding the eigenvalues of the fundamental solution matrix $\mathbf{M}$. To calculate $\mathbf{M}$ and hence to check the stability of the system, we need to find the values of state vectors at the beginning of the cycle $\mathbf{X}(0)$, at the switching $\mathbf{X}\left(d_{1} T\right), \mathbf{X}\left(d_{2} T\right)$ and also the duty cycles $d_{1}, d_{2}$. This can be calculated by numerically solving a nonlinear equation [4], [5]. The computed loci of the eigenvalues with varying input voltage are shown in Fig. 5. The system loses stability through a smooth period doubling bifurcation at input voltage of around $56.7 \mathrm{~V}$. This result is in very good agreement with the simulation results presented in Fig. 2.

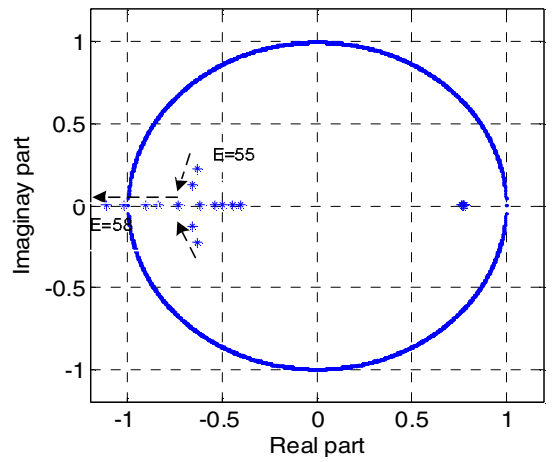

Figure 5. Loci of the eigenvalues of the monodromy matrix for different input voltages

\section{B. Border collision analysis:}

As shown in Fig. 3, a border collision period doubling bifurcation occurs when the sharing current ratio is increased beyond a certain value. In addition, as $m$ increases, the system goes from a situation where one control signal hits the ramp; to another where it just misses the ramp (see Fig. 6). This results in a change in topological sequence. In normal operation, the operation of the system for one switching cycle takes the following sequence: both switches ON, switch $2 \mathrm{ON}$ and switch 1 OFF, finally, both switches OFF. After the border collision occurs, the sequences of the operation are altered to: switch $1 \mathrm{ON}$ and switch $2 \mathrm{OFF}$, both switches ON, switch $2 \mathrm{ON}$ and switch 1 OFF, finally, both switches OFF. This change of the system operation causes the monodromy matrix to alter as well. The new monodromy matrix can be written as:

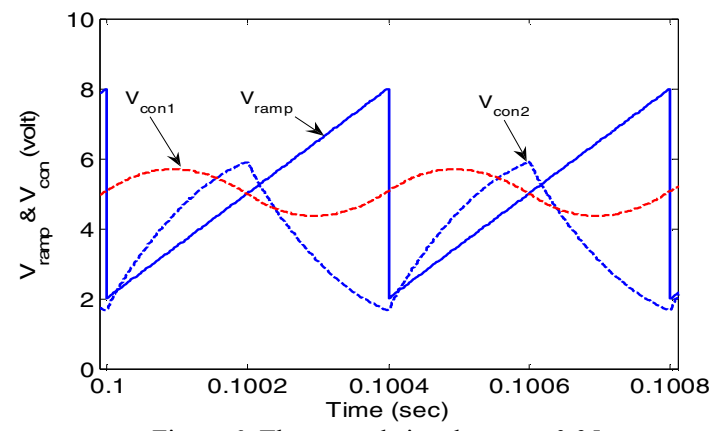

Figure 6 . The control signals at $m=3.25$

$$
\begin{aligned}
\mathbf{M}(0, X(0), T)=e^{\mathbf{A}_{\mathbf{s}}\left(1-d_{2}\right) T} \times \mathbf{S}_{\mathbf{2}} \times e^{\mathbf{A}_{\mathbf{s}}\left(d_{2}-d_{1}\right) T} & \times \mathbf{S}_{\mathbf{1}} \times e^{\mathbf{A}_{\mathbf{s}}\left(d_{1}-d_{3}\right) T} \\
& \times \mathbf{S}_{\mathbf{3}} \times e^{\mathbf{A}_{\mathbf{s}}\left(d_{3}\right) T}
\end{aligned}
$$

where $\mathbf{S}_{\mathbf{3}}, \mathbf{S}_{\mathbf{2}}$ and $\mathbf{S}_{\mathbf{1}}$ are the saltation matrices at $d_{3} T, d_{2} T$ and $d_{1} T$, respectively, $d_{3} T$ is the time when switch 1 turns ON and switch 2 turns OFF. At this time, the indicator function $h\left(X\left(d_{3} T\right)\right)$ is defined by the equation:

$V_{\text {offset }}-K_{p_{2}}\left(x_{1}\left(d_{3} T\right)-V_{\text {ref }}\right)-K i\left(x_{3}\left(d_{3} T\right)-m x_{2}\left(d_{3} T\right)\right)-V_{\text {ramp }}=0$,

$\mathbf{n}=\left[\begin{array}{lll}-K_{p_{2}} & m K_{i} & -K_{i}\end{array}\right]^{T}, \mathbf{f}_{-}\left(x\left(d_{3} T\right)\right)=\mathbf{A}_{\mathbf{s}} \mathbf{x}+\mathbf{B}_{\mathbf{2}} E$ and

$\mathbf{f}_{+}\left(x\left(d_{3} T\right)\right)=\mathbf{A}_{\mathbf{s}} \mathbf{x}+\mathbf{B}_{1} E$.

The saltation matrix $\mathbf{S}_{\mathbf{3}}$ can be calculated from equation (6). The monodromy matrix of the system can be calculated after obtaining the duty ratios semi-analytically. The eigenvalues of $\mathbf{M}$ for different values of the current ratio $m$ are shown in Fig. 7. As the current ratio is increased, the eigenvalues jump abruptly from complex conjugate values to negative real values and one of them lands outside of the unit cycle. This causes a nonsmooth period doubling bifurcation.

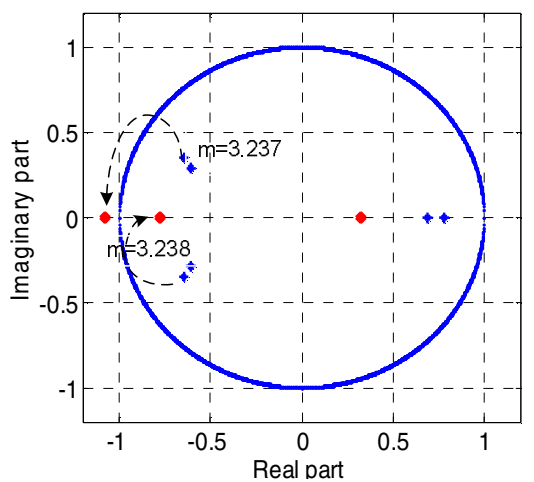

Figure 7. Loci of the eigenvalues of the monodromy matrix for different current ratios

\section{CONTROL OF THE PERIOD DOUBLING BIFURCATION}

We note that stability of the system is governed by the eigenvalues of the state transition matrix over a clock cycle, and this matrix is in turn influenced by the state transition matrices across the switching events. We seek to develop control techniques based on the expression of the saltation matrix (6), so that the eigenvalues of the monodromy matrix remain within the unit circle.

\section{1) Control based on the change of the upper limit of the ramp:}

The system can be stabilized and the eigenvalues of the monodromy matrix can be pushed inside the unit circle by changing the upper value of the $\operatorname{ramp}\left(V_{U}\right)$ to $\left(a \times V_{U}\right)$ with the value of $a \psi$ chosen to determine the desired location. The effect of this change is to alter the time derivative of $h$ hence influencing the saltation matrices (since $\mathbf{S}_{\mathbf{1}}$ and $\mathbf{S}_{\mathbf{2}}$ are functions of the time 
derivative of $h$ ). To optimally design the controller we can numerically solve the following nonlinear transcendental equation

$$
|e i g(\mathbf{M}(T, 0))|-0.64=0
$$

to locate the eigenvalues at a circle of any chosen radius (in this case 0.64) indicating a stable period one limit cycle. Corresponding values of $a$ for different values of input voltage may then be calculated as shown in Fig. 8. The response of the system while the input voltage changes suddenly from 55 to $60 \mathrm{~V}$ (at time $0.1 \mathrm{Sec}$ ) is shown in Fig. 9. It is clear that the system, after a very small transient, will settle down quickly to the stable period 1 limit cycle. Note that the system is unstable at an input voltage of $60 \mathrm{~V}$ without the proposed control.

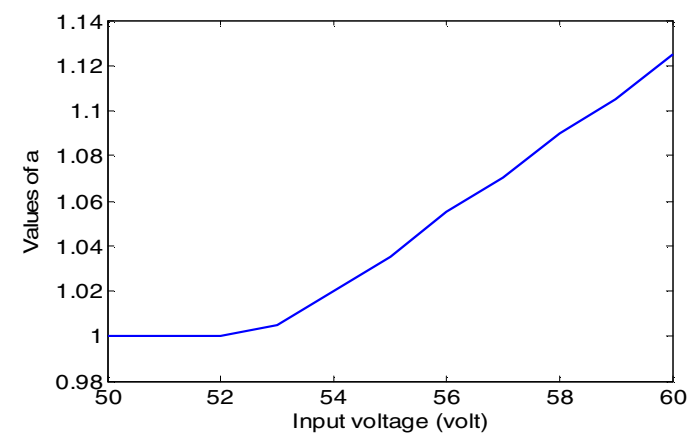

Figure 8. Optimum values of $a$ for first controller

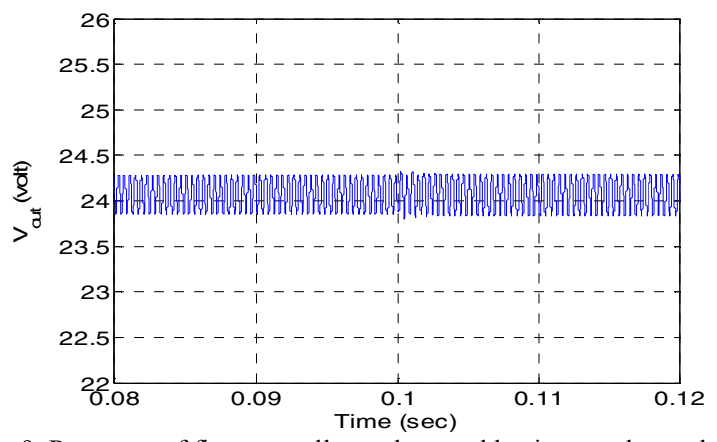

Figure 9. Response of first controller under a sudden input voltage change

\section{2) Control based on additional control signal proportional to the output voltage:}

Another control method is based on changes of the normal to the switching manifold that appears in (6). In this method we slightly change the slope of $h \psi$ to:

$$
h\left(X\left(d_{1} T\right)\right)=V_{\text {offset }}-K_{p 1}\left(x_{1}\left(d_{1} T\right)-V_{\text {ref }}+a x_{1}\left(d_{1} T\right)\right)-V_{\text {ramp }}=0 \text {. }
$$

This gives a normal vector $\left[\begin{array}{lll}-K_{p_{1}}(1+a) & 0 & 0\end{array}\right]^{T}$, which will have an influence on the saltation matrix $\mathbf{S}_{\mathbf{1}}$ (since $\mathbf{S}_{\mathbf{1}}$ is function of the normal vector $\mathbf{n}$ ).

It is obvious that the monodromy matrix of the system will be function of $a$, and thus it is possible to stabilise the period one limit cycle by altering the value of $a$. Again we can locate the eigenvalues at a circle of any chosen radius. The relation between the change of the input voltages and the required values of $a$ for a radius of 0.64 are shown in Fig. 10.

The response of the system when the input voltage changes suddenly from 55 to $60 \mathrm{~V}$ is shown in Fig. 11. In this case also, the system settles to the stable period 1 limit cycle after a brief transient.

Comparison of the two controllers reveals that both controllers extend the period one limit cycle, however, the first controller based on the change of the upper limit of the ramp signal has a smaller steady state error.

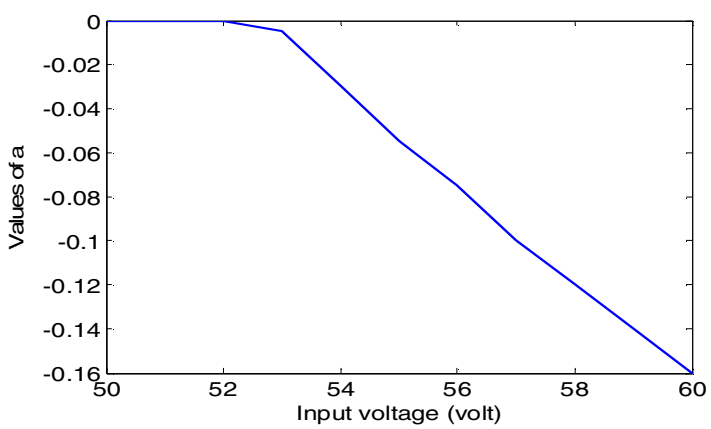

Figure 10 Optimum values of $a$ for second controller

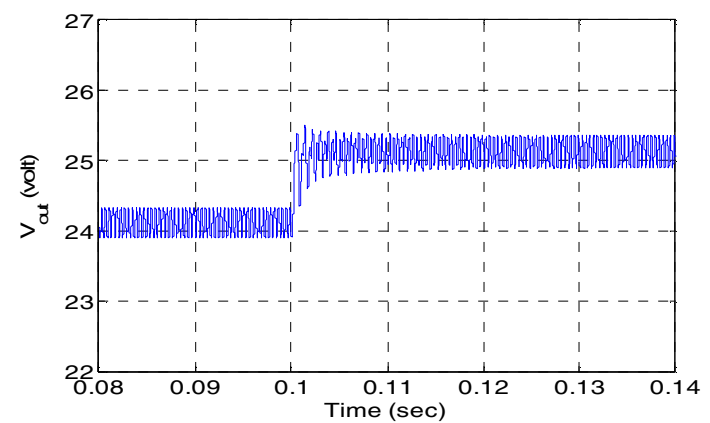

Figure 11. Response of second controller as the input voltage is changed

\section{CONCLUSION}

In the parallel connected buck converter under master-slave current sharing scheme, the normal period-1 operating mode can lose stability through smooth as well as nonsmooth period doubling bifurcations. We have demonstrated the stability analysis of the system using the complete-cycle solution matrix (the monodromy matrix) showing that this matrix can change abruptly if a periodic solution touches a non-smooth hyper-surface. Based on the expression of the saltation matrices, we have proposed and demonstrated two controllers to control the period doubling bifurcation. The first one is based on modifying the upper limit of the controller ramp signal and the second one is based on using an additional control signal proportional to the output voltage. Both controllers provide good results without any complicated bifurcation control law.

\section{REFERENCES}

[1] C. K. Tse, Complex Behavior of Switching Power Converters. Boca Raton, USA: CRC Press, 2003

[2] H. H. c. Iu and C. K. Tse, 'Bifurcation behaviour of parallel connected buck converter'. IEEE Trans. Circ. Syst - I, vol. 48, no. 2, pp.2333-240, 2001.

[3] S. Banerjee and G. C. Verghese, eds., Nonlinear Phenomena in Power Electronics: Attractors, Bifurcations, Chaos, and Nonlinear Control. New York, USA: IEEE Press, 2001.

[4] D. Giaouris, S. Banerjee, B. Zahawi, and V. Pickert, "Stability Analysis of the Continuous Conduction Mode Buck Converter via Filippov's Method," IEEE Transactions on Circuits and Systems - I, 2007 (in Press).

[5] D. Giaouris, A. Elbkosh, V. Pickert, B. Zahawi, and S. Banerjee, "Control of period doubling bifurcations in dc-dc converters," in International Conference Control 2006, (Glasgow - Scotland), 2006.

[6] R. I. Leine and H. Nijmeijer, Dynamics and Bifurcations of NonSmooth Mechanical Systems. Springer, 2004.

[7] R. I. Leine, D. H. V. Campen, and B. L. V. de Vrande, "Bifurcations in nonlinear discontinuous systems," Nonlinear Dynamics, vol. 23, pp. $105-164,2000$.

[8] Giaouris D, Elbkosh A, Banerjee S, Zahawi B, Pickert V. "Control of switching circuits using complete-cycle solution matrices". IEEE International Conference on industrial Technology (ICIT), Mumbai, India, December 2006 